\title{
ON A RANDOM-COEFFICIENT AR(1) PROCESS WITH HEAVY-TAILED RENEWAL SWITCHING COEFFICIENT AND HEAVY-TAILED NOISE
}

\author{
REMIGIJUS LEIPUS, ${ }^{* * *}$ \\ VYGANTAS PAULAUSKAS **** AND \\ DONATAS SURGAILIS, ${ }^{* * * *}$ Vilnius University and \\ Institute of Mathematics and Informatics, Vilnius
}

\begin{abstract}
We discuss the limit behavior of the partial sums process of stationary solutions to the (autoregressive) $\mathrm{AR}(1)$ equation $X_{t}=a_{t} X_{t-1}+\varepsilon_{t}$ with random (renewalreward) coefficient, $a_{t}$, taking independent, identically distributed values $A_{j} \in[0,1]$ on consecutive intervals of a stationary renewal process with heavy-tailed interrenewal distribution, and independent, identically distributed innovations, $\varepsilon_{t}$, belonging to the domain of attraction of an $\alpha$-stable law $(0<\alpha \leq 2, \alpha \neq 1)$. Under suitable conditions on the tail parameter of the interrenewal distribution and the singularity parameter of the distribution of $A_{j}$ near the unit root $a=1$, we show that the partial sums process of $X_{t}$ converges to a $\lambda$-stable Lévy process with index $\lambda<\alpha$. The paper extends the result of Leipus and Surgailis (2003) from the case of finite-variance $X_{t}$ to that of infinitevariance $X_{t}$.
\end{abstract}

Keywords: AR(1) model; regime switching; renewal-reward process; stable Lévy process

2000 Mathematics Subject Classification: Primary 60F05; 60G52

Secondary 60K05

\section{Introduction and main result}

There is a growing econometrics literature on regime switching models that offer an attractive explanation of the long memory and heavy tails observed in financial series. Various regime switching models leading to the long-memory property and related econometrical issues were discussed in Parke (1999), Liu (2000), Jensen and Liu (2006), Gourieroux and Jasiak (2001), Diebold and Inoue (2001), Leipus and Viano (2003), Davidson and Sibbertsen (2005), Granger and Hyung (2004), and Mikosch and Stărică (2004). Recently, Leipus and Surgailis (2003) and Leipus et al. (2005) discussed the random-coefficient (autoregressive) AR(1) equation

$$
X_{t}=a_{t} X_{t-1}+\varepsilon_{t}, \quad t \in \mathbb{Z},
$$

Received 13 April 2004; revision received 29 December 2005.

* Postal address: Department of Mathematics and Informatics, Vilnius University, Naugarduko 24, LT-03225 Vilnius, Lithuania.

** Email address: remigijus.leipus@maf.vu.lt

*** Email address: vygantas.paulaskas@maf.vu.lt

**** Postal address: Institute of Mathematics and Informatics, Akademijos str. 4, LT-08663 Vilnius, Lithuania. Email address: sdonatas@ktl.mii.lt

Supported by bilateral Lithuania-France research project Gilibert and the Lithuanian State Science and Studies Foundation grant T-10/06. 
where $a_{t}$ assumes random values $A_{j} \in[0,1]$ on consecutive intervals $\left(S_{j-1}, S_{j}\right]$ of a renewal process on $\mathbb{Z}$ with a heavy-tailed interrenewal distribution $U_{j}=S_{j}-S_{j-1}$. When the $A_{j}$ assume two values, 0 and 1 , only, the corresponding stationary solution to (1.1) switches between independent, identically distributed (i.i.d.) and random walk (unit-root) regimes, and the autocovariance of $X_{t}$ may decay slowly with the lag, as in fractional autoregressive integrated moving average models (this fact was first observed in Pourahmadi (1988)). The main result of Leipus and Surgailis (2003) says that partial sums of such a regime switching AR(1) model as (1.1) converge to a $\lambda$-stable Lévy process, with index $\lambda<2$, depending on the tail parameter, $\beta$, of the interrenewal distribution $U=U_{j}$ and the singularity parameter, $q$, of the probability density function of generic $A=A_{j}$ near the unit root $a=1$ (see Assumptions $\mathrm{U}(\beta)$ and $\mathrm{A}(q)$, below, for precise definitions of $\beta$ and $q)$. This result is in deep contrast with the Gaussian (fractional Brownian motion) asymptotic distribution of partial sums of finitevariance fractional autoregressive integrated moving average models. Note that the asymptotic stable behavior of partial sums processes of linear models with heavy-tailed renewal switching mean was discussed in Taqqu and Levy (1986), Liu (2000), Davidson and Sibbertsen (2005), Mikosch et al. (2002), Pipiras et al. (2004), and other papers.

The present paper extends the results of Leipus and Surgailis (2003) from the finite-variance case $\left(\mathrm{E}\left[X_{t}^{2}\right]<\infty\right)$ to the infinite-variance case $\left(\mathrm{E}\left[X_{t}^{2}\right]=\infty\right)$. As in the above-mentioned paper, we assume that $\varepsilon_{t}, t \in \mathbb{Z}$, is an i.i.d. sequence and that $a_{t}, t \in \mathbb{Z}$, is a strictly stationary (renewal-reward) process independent of $\varepsilon_{t}, t \in \mathbb{Z}$; i.e.

$$
a_{t}:=A_{j}, \quad S_{j-1}<t \leq S_{j}, j \in \mathbb{Z},
$$

where $S_{j}, j \in \mathbb{Z}$, is a stationary renewal process on $\mathbb{Z}$ with finite-mean interrenewal distribution $U_{j}=S_{j}-S_{j-1}$, and $A_{j}, j \in \mathbb{Z}$, is an i.i.d. sequence independent of $S_{j}, j \in \mathbb{Z}$. Let $\mu:=\mathrm{E}[U]$. Recall that the distribution of the first arrival time, $S_{0} \geq 0$, and the last arrival time, $S_{-1}$, before $t=0$ in a stationary renewal process $\left\{S_{j}\right\}$ satisfy

$$
\mathrm{P}\left[S_{0}=u\right]=\mathrm{P}\left[S_{-1}=-u-1\right]=\mu^{-1} \mathrm{P}[U \geq u+1], \quad u=0,1, \ldots
$$

The following assumptions on the generic distributions $\varepsilon=\varepsilon_{t}, U=U_{j}$, and $A=A_{j}$ play an important role in the asymptotic results for partial sums of $X_{t}$ in (1.1).

Assumption $\mathbf{D}(\alpha)$. (i) If $\alpha=2$ then $\mathrm{E}[\varepsilon]=0$ and $\mathrm{E}\left[\varepsilon^{2}\right]<\infty$.

(ii) If $0<\alpha<2$ then there exist constants $c_{\varepsilon}^{+}, c_{\varepsilon}^{-} \geq 0, c_{\varepsilon}^{+}+c_{\varepsilon}^{-}>0$, such that

$$
\mathrm{P}[\varepsilon>x] \sim c_{\varepsilon}^{+} x^{-\alpha} \text { as } x \rightarrow \infty, \quad \mathrm{P}[\varepsilon<x] \sim c_{\varepsilon}^{-}|x|^{-\alpha} \text { as } x \rightarrow-\infty .
$$

Moreover, $\mathrm{E}[\varepsilon]=0$ for $1<\alpha<2$.

Assumption $\mathbf{U}(\boldsymbol{\beta})$. There exist constants $c_{U}>0$ and $\beta>1$ such that

$$
\mathrm{P}[U=u] \sim c_{U} u^{-\beta-1} \quad \text { as } u \rightarrow \infty .
$$

Assumption A(q). $\mathrm{P}[0 \leq A \leq 1]=1$ and

(i) if $q=0$ then $A$ has an atom at 1 , i.e. $0<f_{1}:=\mathrm{P}[A=1]<1$;

(ii) if $q>0$ then A has a probability density $f(a)$ in some neighborhood of a $=1$ such that

$$
f(a)=f_{1}(a)(1-a)^{q-1},
$$

where $f_{1}(a)$ is a continuous function such that $f_{1}:=f_{1}(1)>0$. 
We shall invoke the above assumptions by writing $\varepsilon \in \mathrm{D}(\alpha), U \in \mathrm{U}(\beta)$, and $A \in \mathrm{A}(q)$, respectively. Note that $\varepsilon \in \mathrm{D}(\alpha)$ means that the random variable (RV) $\varepsilon$ belongs to the domain of normal attraction of a stable law with index $\alpha, 0<\alpha \leq 2$; in other words,

$$
n^{-1 / \alpha} \sum_{i=1}^{n} \varepsilon_{i}-\ell_{n}(\alpha) \stackrel{\mathrm{D}}{\rightarrow} Z,
$$

where $Z$ is an $\alpha$-stable $\mathrm{RV}, \stackrel{\mathrm{D}}{\rightarrow}$ ' denotes convergence in distribution, and $\ell_{n}(\alpha)$ are the centering constants; see Feller (1966, p. 580):

$$
\ell_{n}(\alpha):= \begin{cases}n \mathrm{E}[\sin (\varepsilon / n)], & \alpha=1, \\ 0, & \alpha \neq 1 .\end{cases}
$$

The characteristic function of the RV $Z$ is given by $\mathrm{E}\left[\mathrm{e}^{\mathrm{i} \theta Z}\right]=\exp \left\{-|\theta|^{\alpha} \omega\left(\theta ; \alpha, c_{\varepsilon}^{+}, c_{\varepsilon}^{-}\right)\right\}$, where

$$
\begin{aligned}
& \omega\left(\theta ; \alpha, c_{\varepsilon}^{+}, c_{\varepsilon}^{-}\right) \\
& := \begin{cases}\frac{\Gamma(2-\alpha)}{1-\alpha}\left(\left(c_{\varepsilon}^{+}+c_{\varepsilon}^{-}\right) \cos \left(\frac{\pi \alpha}{2}\right)-\mathrm{i}\left(c_{\varepsilon}^{+}-c_{\varepsilon}^{-}\right) \operatorname{sgn}(\theta) \sin \left(\frac{\pi \alpha}{2}\right)\right), & \alpha \neq 1, \\
\left(c_{\varepsilon}^{+}+c_{\varepsilon}^{-}\right) \frac{\pi}{2}+\mathrm{i}\left(c_{\varepsilon}^{+}-c_{\varepsilon}^{-}\right) \operatorname{sgn}(\theta) \log |\theta|, & \alpha=1 .\end{cases}
\end{aligned}
$$

We shall also need Assumption $\mathrm{D}(\alpha, \delta)$, below, which is a technical assumption stronger than Assumption $\mathrm{D}(\alpha)$ and is sometimes imposed to obtain convergence rates in the central limit theorem (1.4). To formulate it, for $\varepsilon \in \mathrm{D}(\alpha), 0<\alpha<2$, and $r \geq \alpha$, let us define the $r$ th absolute pseudomoment of $\varepsilon$ as

$$
\mu_{r}(\varepsilon):=\int_{\mathbb{R}}|x|^{r}|\mathrm{~d}(\mathrm{P}[\varepsilon \leq x]-\mathrm{P}[Z \leq x])|,
$$

where $Z$ is the $\alpha$-stable RV on the right-hand side of (1.4).

Assumption $\mathrm{D}(\boldsymbol{\alpha}, \boldsymbol{\delta})$. (i) If $\alpha=2$ then $\mathrm{E}[\varepsilon]=0$ and $\mathrm{E}\left[|\varepsilon|^{2+\delta}\right]<\infty$ for some $\delta>0$.

(ii) If $0<\alpha<2$ then $\varepsilon \in \mathrm{D}(\alpha)$ and $\mu_{\alpha+\delta}(\varepsilon)<\infty$ for some $\delta>0$.

We write $\stackrel{\text { FDD }}{\longrightarrow}$ ' to denote weak convergence of finite-dimensional distributions, and define

$$
\lambda:=\frac{\alpha(\beta+q)}{1+\alpha} .
$$

In Theorem 1.1, below, $X_{t}$ is a stationary solution to (1.1) given by

$$
X_{t}=\varepsilon_{t}+\sum_{i=1}^{\infty} \varepsilon_{t-i} \prod_{p=0}^{i-1} a_{t-p} .
$$

For $0<\lambda<2$, introduce a $\lambda$-stable Lévy process $Z_{\lambda}(\tau), \tau \geq 0$, with independent and stationary increments, whose $d$-dimensional characteristic function is given by

$$
\mathrm{E}\left[\exp \left\{\mathrm{i} \sum_{j=1}^{d} \theta_{j} Z_{\lambda}\left(\tau_{j}\right)\right\}\right]=\exp \left\{-\mu^{-1} \sum_{j=1}^{d}\left|\sum_{k=j}^{d} \theta_{k}\right|^{\lambda} \omega\left(\sum_{k=j}^{d} \theta_{k} ; \lambda, c_{Y}^{+}, c_{Y}^{-}\right)\left(\tau_{j}-\tau_{j-1}\right)\right\}
$$


for $\tau_{0}, \ldots, \tau_{d}$ with $0=\tau_{0}<\tau_{1}<\cdots<\tau_{d}$, and $\theta_{j} \in \mathbb{R}, j=1, \ldots, d$, where

$$
c_{Y}^{+}:=c_{V} \mathrm{E}\left[|Z|^{\lambda} \mathbf{1}(Z>0)\right], \quad c_{Y}^{-}:=c_{V} \mathrm{E}\left[|Z|^{\lambda} \mathbf{1}(Z<0)\right] .
$$

In (1.8), $Z$ is the same as in (1.4) and the constant $c_{V} \equiv c_{V}(\alpha, \beta, q)>0$ is given explicitly later, in (3.7). Let $[n \tau]$ denote the integer part of $n \tau$.

Theorem 1.1. Let Assumptions $\mathrm{A}(q), \mathrm{U}(\beta)$, and $\mathrm{D}(\alpha, \delta)$ be satisfied for some $q \geq 0, \beta>1$, $\alpha$ with $0<\alpha \leq 2$ and $\alpha \neq 1$, and $\delta>0$ such that $\beta+q<1+\alpha$. Then

$$
n^{-1 / \lambda} \sum_{0 \leq s<[n \tau]} X_{s} \stackrel{\text { FDD }}{\longrightarrow} Z_{\lambda}(\tau) .
$$

Remark 1.1. It follows from (1.7) and (1.8) that the limit process $Z_{1}(\tau)$ in (1.9), corresponding to $\lambda=1$, is symmetric (i.e. $c_{Y}^{+}=c_{Y}^{-}$), due to the fact that the RV $Z$ in (1.4) has mean 0 for $\alpha>1$. On the other hand, if $\lambda \neq 1$ then the process $Z_{\lambda}(\tau)$ need not be symmetric, since

$$
\mathrm{E}\left[|Z|^{\lambda} \mathbf{1}(Z>0)\right] \neq \mathrm{E}\left[|Z|^{\lambda} \mathbf{1}(Z<0)\right]
$$

in general. The last inequality holds, e.g. if $Z$ is not symmetric and $\lambda$ is sufficiently close to $\alpha$ (Samorodnitsky and Taqqu (1994, p. 19)). The lack of symmetry of $Z_{\lambda}(\tau)$ in Theorem 1.1 contrasts with the limit process labeled $Z_{\lambda}(\tau)$ in Leipus and Surgailis (2003, Theorem 4.1), which is symmetric (in that paper $\alpha=2, \beta \equiv \alpha, \lambda \in\left(\frac{4}{3}, 2\right)$, and $Z \sim N(0,1)$; the symmetry of $\varepsilon$ is not assumed).

Remark 1.2. The case $\alpha=1$ is more delicate and remains open. If the distribution of $\varepsilon$ is symmetric then we expect that the convergence (1.9) also holds in this case, with $\lambda=$ $(\beta+q) / 2 \in(0,1)$, as in (1.5). However, if $\alpha=1$ and $c_{\varepsilon}^{+} \neq c_{\varepsilon}^{-}$then we conjecture that a limit of partial sums of $X_{t}$ exists under the normalization $(n \log n)^{-1 / \lambda}$ rather than under $n^{-1 / \lambda}$, and that the limiting process $Z_{\lambda}(\tau)$ is completely antisymmetric.

Remark 1.3. While positivity of $A$ (see Assumption A $(q)$ ) can probably be weakened, the fact that $|A| \leq 1$ is crucial in Theorem 1.1. The regime in (1.1) corresponding to $a_{t}=a>1$ can be described as 'explosive growth' whose duration should be quite short ( $U$ should have an exponential tail) in order that a stationary $L^{r}$-solution $(r>0)$ exists; see Remark 2.1(iii). See also Leipus et al. (2005) on AR(1) process switching between the i.i.d. regime $a=0$ and some (deterministic) value $A>1$. A particular case of this process is the collapsible bubbles model introduced in Blanchard (1979).

Under the assumptions of Theorem 1.1, the stationary solution $X_{t},(1.6)$, with $a_{t}$ as in (1.2) has finite variance if and only if $\alpha=2$ and $2<\beta+q$. Moreover, for $2<\beta+q<3$ the autocovariance function of $X_{t}$ decays slowly, as $t^{2-\beta-q}$; see Leipus and Surgailis (2003, Theorem 3.1). The last property is usually referred to as long memory. We might also expect some kind of long memory for $X_{t}$ in Theorem 1.1 when $\mathrm{E}\left[X_{t}^{2}\right]=\infty$. In particular, our limit results can be linked to the LRD(SAV) property introduced in Heyde and Yang (1997) and the self-normalization discussed therein. (The possibility of such a connection was suggested by the referee.) On the other hand, the limit process $Z_{\lambda}(\tau)$ in Theorem 1.1 has independent increments, which is an indication of short memory of the summands (see Cox (1984) and Dehling and Philipp (2002)). Note that $\lambda<\alpha$ for any $\alpha \in(0,2]$; in other words, variability of the limit process in (1.9) is strictly greater than variability of the summands. A rather general 
scheme of renewal regime switching that exhibits a similar increase of variability was discussed in Leipus et al. (2005).

The regular asymptotics in Assumptions $\mathrm{A}(q), \mathrm{U}(\beta)$, and $\mathrm{D}(\alpha)$ help to avoid additional technicalities and can probably be generalized to include slowly varying factors. We expect that for $\beta+q>1+\alpha$, Theorem 1.1 holds with $\lambda=\alpha$ (and some constants, $c_{Y}^{+}$and $c_{Y}^{-}$, in (1.7) proportional to $c_{\varepsilon}^{+}$and $c_{\varepsilon}^{-}$); in other words, when $\beta+q>1+\alpha$, partial sums of $X_{t}$ should behave similarly to partial sums of $\varepsilon_{t}$.

The rest of the paper is organized as follows. In Section 2 we discuss the existence of a stationary solution to $X_{t},(1.6)$, in the $L^{r}$-sense $(r>0)$ and the $\operatorname{LRD}(\mathrm{SAV})$ property of $X_{t}$. (LRD and SAV stand for long-range dependence and sample Allen variance, respectively.) Theorem 1.1 is proved in Section 3. Section 4 contains the proofs of the auxiliary results Lemmas 3.1-3.5.

\section{Existence of a stationary solution}

Random-coefficient AR(1) equation (1.1), under general conditions on the $\mathbb{R}^{2}$-valued process $\left(a_{t}, \varepsilon_{t}\right)$, has been studied by many authors; see Vervaat (1979), Brandt (1986), Pourahmadi (1988), Karlsen (1990), and the references therein. Theorem 2.1, below, states conditions for the existence of stationary solution (1.6) with finite $r$ th moment, when $a_{t}$ is a renewal-reward process of the form (1.2). In this theorem, we do not invoke Assumption $\mathrm{A}(q), \mathrm{U}(\beta)$, or $\mathrm{D}(\alpha)$. Let $v_{r}:=\mathrm{E}\left[|A|^{r}\right]$.

Theorem 2.1. Let $r$ and $p, 0<r \leq p \leq 2$, be given, and let $\mathrm{E}\left[|\varepsilon|^{p}\right]<\infty$. Equation (1.1), with $a_{t}$ as in (1.2), admits a stationary solution, $X_{t}$, given by (1.6), with $\mathrm{E}\left[\left|X_{t}\right|^{r}\right]<\infty$ if

$$
\mathrm{E}\left[v_{r U}\right]<1
$$

and

$$
\mathrm{E}\left[\sum_{v=1}^{U}\left\{1+v_{p}+\cdots+v_{p v}\right\}^{r / p}\right]<\infty .
$$

Proof. We shall use the following inequality. Let $0<p \leq 2$ and let $\xi_{1}, \xi_{2}, \ldots$ be random variables with $\mathrm{E}\left[\left|\xi_{i}\right|^{p}\right]<\infty$. Moreover, for $1<p \leq 2$ we assume that the $\mathrm{RVs} \xi_{i}$ form a martingale difference sequence:

$$
\mathrm{E}\left[\xi_{i+1} \mid \xi_{i}, \ldots, \xi_{1}\right]=0, \quad i=1,2, \ldots
$$

There then exists a constant, $C_{p}<\infty$, which depends only on $p$, such that

$$
\mathrm{E}\left[\left|\sum_{i} \xi_{i}\right|^{p}\right] \leq C_{p} \sum_{i} \mathrm{E}\left[\left|\xi_{i}\right|^{p}\right] .
$$

For $0<p \leq 1$, (2.3) holds with $C_{p}=1$, and for $1<p \leq 2$ it holds with $C_{p}=2$ (see von Bahr and Esseen (1965)). The theorem follows if we show that the series for $X_{t}$ in (1.6) converges in $L^{r}$. Clearly, it suffices to consider the case $t=0$. Write $X_{0}=X_{0}^{0}+X_{0}^{1}$, where

$$
X_{0}^{0}:=\sum_{S_{-1}<u \leq 0} A_{0}^{-u} \varepsilon_{u}, \quad X_{0}^{1}:=\sum_{u \leq S_{-1}} a_{0} \cdots a_{u+1} \varepsilon_{u} .
$$


Using (2.3), (1.3), and Jensen's inequality, as in Leipus and Surgailis (2003) we set $c_{1}=$ $\left(2 \mathrm{E}\left[|\varepsilon|^{p}\right]\right)^{r / p}$ and obtain

$$
\begin{aligned}
\mathrm{E}\left[\left|X_{0}^{0}\right|^{r}\right] & \leq \mathrm{E}\left[\mathrm{E}\left[\left|\sum_{S_{-1}<u \leq 0} A_{0}^{-u} \varepsilon_{u}\right|^{p} \mid A_{0}, S_{-1}\right]\right]^{r / p} \\
& \leq c_{1} \mathrm{E}\left[\sum_{S_{-1}<u \leq 0}\left|A_{0}\right|^{-p u}\right]^{r / p} \\
& \leq c_{1} \sum_{v=1}^{\infty}\left\{\sum_{-v<u \leq 0} \mathrm{E}\left[\left|A_{0}\right|^{-p u} \mid S_{-1}=-v\right]\right\}^{r / p} \mathrm{P}\left[S_{-1}=-v\right] \\
& =c_{1} \mu^{-1} \mathrm{E}\left[\sum_{v=1}^{U}\left\{1+v_{p}+\cdots+v_{p(v-1)}\right\}^{r / p}\right] .
\end{aligned}
$$

Next, consider $\mathrm{E}\left[\left|X_{0}^{1}\right|^{r}\right]$. As $X_{0}^{1}=A_{0}^{-S_{-1}} \sum_{u \leq S_{-1}} a_{S_{-1}} \cdots a_{u+1} \varepsilon_{u}$, we have

$$
\mathrm{E}\left[\left|X_{0}^{1}\right|^{r}\right]=\sum_{v=1}^{\infty} \mathrm{E}\left[\left|A_{0}\right|^{r v}\left|\sum_{u \leq-v} a_{-v} \cdots a_{u+1} \varepsilon_{u}\right|^{r} \mid S_{-1}=-v\right] \mathrm{P}\left[S_{-1}=-v\right] .
$$

Since $A_{0}$ is independent of $A_{-1}, A_{-2}, \ldots, S_{-1}, S_{-2}, \ldots$ and the $\varepsilon_{u}$, we have

$$
\begin{aligned}
& \mathrm{E}\left[\left|A_{0}\right|^{r v}\left|\sum_{u \leq-v} a_{-v} \cdots a_{u+1} \varepsilon_{u}\right|^{r} \mid S_{-1}=-v\right] \\
& =\mathrm{E}\left[\left|A_{0}\right|^{r v}\right] \mathrm{E}\left[\left|\sum_{u \leq-v} a_{-v} \cdots a_{u+1} \varepsilon_{u}\right|^{r} \mid S_{-1}=-v\right] .
\end{aligned}
$$

Hence,

$$
\begin{aligned}
\mathrm{E}\left[\left|X_{0}^{1}\right|^{r}\right] & =\sum_{v=1}^{\infty} \mathrm{P}\left[S_{-1}=-v\right] v_{r v} \mathrm{E}\left[\left|\sum_{u \leq-v} a_{-v} \cdots a_{u+1} \varepsilon_{u}\right|^{r} \mid S_{-1}=-v\right] \\
& =\sum_{v=1}^{\infty} \mathrm{P}\left[S_{-1}=-v\right] v_{r v} \mathrm{E}\left[\left|\sum_{u \leq 0} a_{0} a_{-1} \cdots a_{u+1} \varepsilon_{u}\right|^{r} \mid S_{0}=0\right] \\
& =c_{2} J
\end{aligned}
$$

where

$$
c_{2}:=\sum_{v=1}^{\infty} \mathrm{P}\left[S_{-1}=-v\right] v_{r v}=\mu^{-1} \sum_{v=1}^{\infty} v_{r v} \mathrm{P}[U \geq v]=\mu^{-1} \mathrm{E}\left[\sum_{u=1}^{U} v_{r u}\right]
$$

and

$$
J:=\mathrm{E}\left[\left|\sum_{u \leq 0} a_{0} a_{-1} \cdots a_{u+1} \varepsilon_{u}\right|^{r} \mid S_{0}=0\right]=\mathrm{E}\left[\left|X_{0}\right|^{r} \mid S_{0}=0\right] .
$$

Note that $c_{2}$ is finite, due to $v_{r u} \leq v_{p u}^{r / p} \leq\left\{1+v_{p}+\cdots+v_{p u}\right\}^{r / p}$ and (2.2).

The conditional expectation $J=\mathrm{E}\left[\left|X_{0}\right|^{r} \mid S_{0}=0\right]$ can be estimated similarly to $\mathrm{E}\left[\left|X_{0}\right|^{r}\right]$ : the only difference is in using the conditional distribution $\mathrm{P}\left[S_{-1}=-v \mid S_{0}=0\right]=\mathrm{P}[U=v]$. 
We shall also need the following inequality: for any real numbers $a, b \in \mathbb{R}$, any $r, 0<r \leq 2$, and any $\delta>0$, we have

$$
|a+b|^{r} \leq(1+\delta)|a|^{r}+\left(1+\delta^{-1}\right)|b|^{r} .
$$

Using (2.6), we can write

$$
\begin{aligned}
J & \leq\left(1+\delta^{-1}\right) \mathrm{E}\left[\left|\sum_{S_{-1}<u \leq 0} A_{0}^{-u} \varepsilon_{u}\right|^{r} \mid S_{0}=0\right]+(1+\delta) \mathrm{E}\left[\left|\sum_{u \leq S_{-1}} a_{0} \cdots a_{u+1} \varepsilon_{u}\right|^{r} \mid S_{0}=0\right] \\
& =:\left(1+\delta^{-1}\right) J_{0}+(1+\delta) J_{1} .
\end{aligned}
$$

Here, as in (2.4),

$$
\begin{aligned}
J_{0} & \leq c_{1} \sum_{v=1}^{\infty}\left\{\sum_{u=0}^{v-1} v_{p u}\right\}^{r / p} \mathrm{P}\left[S_{-1}=-v \mid S_{0}=0\right] \\
& =c_{1} \sum_{v=1}^{\infty}\left\{\sum_{u=0}^{v-1} v_{p u}\right\}^{r / p} \mathrm{P}[U=v] \\
& =c_{1} \mathrm{E}\left[\left\{\sum_{u=0}^{U-1} v_{p u}\right\}^{r / p}\right] .
\end{aligned}
$$

Also, as in (2.5),

$$
J_{1}=J \sum_{v=1}^{\infty} v_{r v} \mathrm{P}\left[S_{-1}=-v \mid S_{0}=0\right]=J \sum_{v=1}^{\infty} v_{r v} \mathrm{P}[U=v]=J \mathrm{E}\left[v_{r U}\right] .
$$

We now have

$$
J \leq c_{1}\left(1+\delta^{-1}\right) \mathrm{E}\left[\left\{\sum_{u=0}^{U-1} v_{p u}\right\}^{r / p}\right]+J(1+\delta) \mathrm{E}\left[v_{r U}\right] .
$$

Condition (2.1) implies that $(1+\delta) \mathrm{E}\left[v_{r U}\right]<1$ for a small enough $\delta>0$, resulting in

$$
J \leq \frac{c_{1}\left(1+\delta^{-1}\right) \mathrm{E}\left[\left\{\sum_{u=0}^{U-1} v_{p u}\right\}^{r / p}\right]}{1-(1+\delta) \mathrm{E}\left[v_{r}\right]}
$$

By combining (2.4)-(2.8), we obtain

$$
\begin{aligned}
\mathrm{E}\left[\left|X_{0}\right|^{r}\right] & \leq 2\left(\mathrm{E}\left[\left|X_{0}^{0}\right|^{r}\right]+\mathrm{E}\left[\left|X_{0}^{1}\right|^{r}\right]\right) \\
& \leq 2 c_{1}\left(\mu^{-1} \mathrm{E}\left[\sum_{u=1}^{U}\left\{\sum_{i=0}^{u-1} v_{p i}\right\}^{r / p}\right]+\frac{\left(1+\delta^{-1}\right) c_{2} \mathrm{E}\left[\left\{\sum_{u=0}^{U-1} v_{p u}\right\}^{r / p}\right]}{1-(1+\delta) \mathrm{E}\left[v_{r U}\right]}\right) .
\end{aligned}
$$

Theorem 2.1 is thus proved.

Remark 2.1. (i) In the case $p=r=2$, conditions (2.1) and (2.2) are also necessary for the existence of a stationary $L^{2}$-solution; see Leipus and Surgailis (2003, Theorem 2.1).

(ii) Condition (2.1) is satisfied if $\mathrm{P}[|A| \leq 1]=1$ and $\mathrm{P}[|A| \neq 1]>0$. Under Assumptions $\mathrm{A}(q)$, $\mathrm{U}(\beta)$, and $\mathrm{D}(\alpha),(2.2)$ can easily be specified in terms of parameters $q, \beta$, and $\alpha$. In particular, for $A \in \mathrm{A}(q), U \in \mathrm{U}(\beta), \varepsilon \in \mathrm{D}(\alpha), 0<\alpha<2$, and $0 \leq q<1$, (2.2) holds for any $r$ and $p$ with $0<r<p(\beta-1) /(1-q)$ and $r \leq p<\alpha$. A similar result also easily follows in the case $\alpha=2$. 
(iii) If $\mathrm{P}[|A|>1]>0$ then $\mathrm{E}\left[v_{r}\right]<\infty$ implies that $\mathrm{P}[U>u]=O\left(\mathrm{e}^{-c u}\right)($ as $u \rightarrow \infty)$ for some $c=c(r)>0$. As long as (2.1) and (2.2) hold, a stationary $L^{r}$-solution $X_{t}$ always exists (Theorem 2.1), although in the case of 'explosive growth' $(\mathrm{P}[|A|>1]>0)$, it will have short duration, since then $U$ has an exponentially decreasing tail.

Heyde and Yang (1997) introduced a notion of long-range dependence based on selfnormalization, which does not require finite variance. Namely, a stationary zero-mean process $X_{t}$ is said to have $\mathrm{LRD}(\mathrm{SAV})$ if

$$
\frac{\left(\sum_{t=1}^{n} X_{t}\right)^{2}}{\sum_{t=1}^{n} X_{t}^{2}} \stackrel{\mathrm{P}}{\rightarrow} \infty
$$

where ' $\stackrel{\text { P }}{\rightarrow}$ ' stands for convergence in probability. It is known (see, e.g. Chistyakov and Götze (2004)) that in the case of an i.i.d. $X_{t}=\varepsilon_{t}$ satisfying Assumption $\mathrm{D}(\alpha)$ (with $0<\alpha \leq 2$ and $\alpha \neq 1$ ), the quotient in (2.9) has a (proper) limit distribution and the $\mathrm{LRD}(\mathrm{SAV})$ property does not hold.

In the case when the $X_{t}$ are of the form (1.1), we obtain the following result.

Corollary 2.1. Let $X_{t}$ satisfy the assumptions of Theorem 1.1, with $1<\alpha \leq 2$. In addition, assume that either $\beta+q>2$ or $\beta+q \leq 2$ and $(\beta-1) /(1-q)>(\beta+q) /(1+\alpha)$. Then $X_{t}$ has $\operatorname{LRD}(S A V)$.

Proof. In view of the statement of Theorem 1.1, it suffices to show that $\sum_{t=1}^{n} X_{t}^{2}=o_{p}\left(n^{2 / \lambda}\right)$, or $\mathrm{E}\left[\left|\sum_{t=1}^{n} X_{t}^{2}\right|^{r / 2}\right]=o\left(n^{r / \lambda}\right)$ for some $r>0$. By stationarity and (2.3),

$$
\mathrm{E}\left[\left|\sum_{t=1}^{n} X_{t}^{2}\right|^{r / 2}\right] \leq \mathrm{E}\left[\left|X_{0}\right|^{r} n\right]=o\left(n^{r / \lambda}\right)
$$

provided that $r$ satisfies $\lambda<r \leq 2$ and $\mathrm{E}\left[\left|X_{0}\right|^{r}\right]<\infty$. From Theorem 2.1 and Remark 2.1(ii), we see that such an $r$ exists if either $(\beta-1) /(1-q)>1$ (in this case we can take $r=p$ to be arbitrarily close to $\alpha$, whence $r>\lambda$ ) or

$$
\frac{\beta-1}{1-q} \leq 1 \quad \text { and } \quad \lambda<\alpha \frac{\beta-1}{1-q} .
$$

(In the latter case we can take any $r, \lambda<r<\alpha(\beta-1) /(1-q)$, and then take $p<\alpha$ sufficiently close to $\alpha$. Also note that, for $q=0$, the second inequality in (2.10) implies that $\lambda>1$.) The corollary is thus proved.

\section{Proof of Theorem 1.1}

Let $S_{-}(t)$ be the last renewal time before time $t$ :

$$
S_{-}(t):=\max \left\{S_{j}: S_{j}<t\right\}
$$

Then $X_{t}=X_{t}^{1}+X_{t}^{0}$, where

$$
X_{t}^{0}:=\sum_{S_{-}(t)<s \leq t} a_{t} \cdots a_{s+1} \varepsilon_{s}, \quad X_{t}^{1}:=\sum_{s \leq S_{-}(t)} a_{t} \cdots a_{s+1} \varepsilon_{s} .
$$

In Lemmas 3.1-3.3 we assume that the conditions of Theorem 1.1 are satisfied. The proofs of all auxiliary lemmas in this section are relegated to Section 4. 
Lemma 3.1. For any $r<\alpha$ sufficiently close to $\alpha$, there exists a $\theta \equiv \theta(r, \alpha, \beta, q)>\lambda$ such that

$$
\mathrm{E}\left[\left|\sum_{t=1}^{n} X_{t}^{1}\right|^{r}\right]=O\left(n^{r / \theta}\right)
$$

According to Lemma 3.1, the component $X_{t}^{1}$ is negligible in the limit (1.9), and we can replace $X_{t}$ in Theorem 1.1 by $X_{t}^{0}$. As in Leipus and Surgailis (2003), write $\sum_{0 \leq s<[n \tau]} X_{s}^{0}=$ $\sum_{1 \leq i \leq N_{[n \tau]-1}} Y_{i}+R_{n}$, where

$$
N_{t}:=\max \left\{j \geq 0: S_{j} \leq t\right\}
$$

i.e. $N_{t}+1$ is the number of renewal points in the interval $[0, t]$, and

$$
Y_{i}:=\sum_{S_{i-1}<s \leq S_{i}}\left(\varepsilon_{s}+A_{i} \varepsilon_{s-1}+\cdots+A_{i}^{s-S_{i-1}-1} \varepsilon_{S_{i-1}+1}\right), \quad i=1,2, \ldots,
$$

is the sum of the $\mathrm{AR}(1)$ processes with parameter $A_{i}$ in the renewal interval $\left[S_{i-1}+1, S_{i}\right]$. By $R_{n}$ we denote the corresponding sum in two extreme subintervals,

$$
\left[0, S_{0}\right] \quad \text { and }\left[S_{N_{[n \tau]-1}}+1,[n \tau]-1\right],
$$

of the interval $[0,[n \tau]-1]$. This can easily be shown to be bounded in probability: $R_{n}=O_{p}(1)$. The proof of Theorem 1.1 therefore reduces to the following relation:

$$
n^{-1 / \lambda} \sum_{i=1}^{N_{[n \tau]}} Y_{i} \stackrel{\mathrm{FDD}}{\longrightarrow} Z_{\lambda}(\tau)
$$

Lemma 3.2. We have $\sum_{1 \leq i \leq N_{[n \tau]}} Y_{i}-\sum_{1 \leq i \leq[n \tau / \mu]} Y_{i}=o_{p}\left(n^{1 / \lambda}\right)$.

By the above lemma, (3.2) and, hence, Theorem 1.1 follow from

$$
n^{-1 / \lambda} \sum_{i=1}^{[n \tau / \mu]} Y_{i} \stackrel{\mathrm{FDD}}{\longrightarrow} Z_{\lambda}(\tau)
$$

Let

$$
\begin{aligned}
& T(a, n):=\sum_{s=1}^{n}\left(\varepsilon_{s}+a \varepsilon_{s-1}+\cdots+a^{s-1} \varepsilon_{1}\right), \\
& \Phi(a, n):=1^{\alpha}+(1+a)^{\alpha}+\cdots+\left(1+a+\cdots+a^{n-1}\right)^{\alpha}, \\
& Z(a, n):=\frac{T(a, n)}{\Phi^{1 / \alpha}(a, n)} .
\end{aligned}
$$

Observe that the $Y_{i}$ are conditionally independent given $S_{j}$ and $A_{j}, j \in \mathbb{Z}$, and

$$
\operatorname{Law}\left(Y_{i} \mid S_{j}, A_{j}, j \in \mathbb{Z}\right)=\operatorname{Law}\left(T\left(A_{i}, U_{i}\right)\right) .
$$

As $\left(A_{i}, U_{i}\right), i \geq 1$, are independent, this implies that $Y_{i}, i \geq 1$, are i.i.d. RVs, with generic distribution $Y \stackrel{\overline{\mathrm{D}}}{=} \Phi^{1 / \alpha}(A, U) Z(A, U)=T(A, U)$, where $\stackrel{\text { ' }}{=}$ ' denotes equality in distribution. 
Lemma 3.3. We have

$$
\mathrm{P}[Y>x] \sim c_{Y}^{+} x^{-\lambda} \text { as } x \rightarrow \infty, \quad \mathrm{P}[Y \leq x] \sim c_{Y}^{-}|x|^{-\lambda} \text { as } x \rightarrow-\infty,
$$

where $c_{Y}^{+}$and $c_{Y}^{-}$are as defined in (1.8). Moreover, if $\lambda=1$ then

$$
\lim _{n \rightarrow \infty} n \mathrm{E}[\sin (Y / n)]=0 .
$$

From Lemma 3.3 and the classical central limit theorem (cf. (1.4)), we have the convergence in (3.3), where $Z_{\lambda}(\tau), \tau \geq 0$, is the Lévy process defined in Theorem 1.1. This concludes the proof of Theorem 1.1.

The proof of Lemma 3.3 uses a generalization of Breiman's lemma (Lemma 4.1, below) for 'tail-independent' RVs $\Phi(A, U)$ and $Z(A, U)$, together with Lemmas 3.4 and 3.5.

Lemma 3.4. We have

$$
\mathrm{P}[\Phi(A, U)>x] \sim c_{V} x^{-\lambda / \alpha} \text { as } x \rightarrow \infty
$$

where

$$
c_{V}:= \begin{cases}c_{U} f_{1}(1+\alpha)^{-\beta /(1+\alpha)} \beta^{-1}, & q=0, \\ c_{U} f_{1} \int_{0}^{\infty} \frac{\mathrm{d} y}{y^{1+\beta+q}} \int_{0}^{\infty} \frac{\mathrm{d} v}{v^{1-q}} \mathbf{1}\left(y^{1+\alpha} \Theta(v)>1\right), & q>0,\end{cases}
$$

and

$$
\Theta(v):=v^{-\alpha} \int_{0}^{1}\left(1-\mathrm{e}^{-v \tau}\right)^{\alpha} \mathrm{d} \tau, \quad v>0 .
$$

Lemma 3.5. We have

$$
\lim _{n \rightarrow \infty} \sup _{a \in[0,1]} \sup _{x \in \mathbb{R}}|\mathrm{P}[Z(a, n) \leq x]-\mathrm{P}[Z \leq x]|=0 .
$$

Moreover, there exists a constant, $C<\infty$, such that, for sufficiently large $n$ and $x>0$,

$$
\sup _{0 \leq a \leq 1} \mathrm{P}[|Z(a, n)|>x] \leq C x^{-\alpha} .
$$

\section{Proofs of Lemmas 3.1-3.5}

A generic constant, $C$, will be used in the proofs below to represent positive numbers whose precise values are not required.

Proof of Lemma 3.1. We have

$$
\sum_{t=1}^{n} X_{t}^{1}=\sum_{s \leq n-1}\left\{\sum_{t=1 \vee(s+1)}^{n} a_{t} \cdots a_{s+1} \mathbf{1}\left(s \leq S_{-}(t)\right)\right\} \varepsilon_{s} .
$$

By (2.3), for any $r, 0<r<\alpha$,

$$
\mathrm{E}\left[\left|\sum_{t=1}^{n} X_{t}^{1}\right|^{r}\right] \leq c_{3} \sum_{s \leq n-1} \mathrm{E}\left[\left\{\sum_{t=1 \vee(s+1)}^{n} a_{t} \cdots a_{s+1} \mathbf{1}\left(s \leq S_{-}(t)\right)\right\}^{r}\right],
$$


where $c_{3}=2 \mathrm{E}\left[|\varepsilon|^{r}\right]<\infty$, a constant. Next, applying the Minkowski inequality (for $r>1$ ) and the inequality $|a+b|^{r} \leq|a|^{r}+|b|^{r}$ (for $0<r \leq 1$ ) yields

$$
\mathrm{E}\left[\left|\sum_{t=1}^{n} X_{t}^{1}\right|^{r}\right] \leq \begin{cases}C \sum_{s \leq n-1}\left\{\sum_{t=1 \vee(s+1)}^{n} \gamma_{t-s}^{1 / r}\right\}^{r} & \text { if } 1<r<\alpha, \\ C \sum_{s \leq n-1} \sum_{t=1 \vee(s+1)}^{n} \gamma_{t-s} & \text { if } 0<r \leq 1,\end{cases}
$$

where

$$
\gamma_{t-s}:=\mathrm{E}\left[a_{t}^{r} \cdots a_{s+1}^{r} \mathbf{1}\left(s \leq S_{-}(t)\right)\right] \leq C(t-s)^{-q-\beta}, \quad s<t .
$$

Indeed, for $r \geq 1$, we have

$$
\mathrm{E}\left[a_{t}^{r} \cdots a_{s+1}^{r} \mathbf{1}\left(s \leq S_{-}(t)\right)\right] \leq \mathrm{E}\left[a_{t} \cdots a_{s+1} \mathbf{1}\left(s \leq S_{-}(t)\right)\right]
$$

and the bound (4.2) follows from Leipus and Surgailis (2003, pp. 743-744). For $0<r<1$, (4.2) follows similarly.

To prove (3.1), first consider the case $1<r<\alpha$. Then, by (4.1) and (4.2),

$$
\mathrm{E}\left[\left|\sum_{t=1}^{n} X_{t}^{1}\right|^{r}\right] \leq C\left(\sum_{|s|<n}\left\{\sum_{t=1}^{n} t^{-(q+\beta) / r}\right\}^{r}+\sum_{s \geq n}\left\{\sum_{t=1}^{n}(t+s)^{-(q+\beta) / r}\right\}^{r}\right)=: C\left(I_{1}+I_{2}\right) .
$$

If $q+\beta>r$ then $I_{1}=O(n)$, implying that $I_{1}=O\left(n^{r / \theta}\right)$ for $r<\alpha$ sufficiently close to $\alpha$ and $\theta>\lambda$ sufficiently close to $\lambda$. The case in which $q+\beta=r$ follows similarly. If $q+\beta<r$ then $I_{1} \leq C n^{1+r-q-\beta}$, meaning that $I_{1}=O\left(n^{r / \theta}\right)$ follows, for some $\theta>\lambda$, from

$$
1+r-q-\beta<\frac{r(1+\alpha)}{\alpha(\beta+q)} \text {. }
$$

It suffices to show (4.3) for $r=\alpha$ (because then it is also satisfied for all $r<\alpha$ sufficiently close to $\alpha$ ), in which case (4.3) becomes $1+\alpha-q-\beta<(1+\alpha) /(\beta+q)$ or, equivalently, $(1+\alpha)(1-1 /(\beta+q))<\beta+q$. However, $1+\alpha \leq 3$ and, so, (4.3) follows from $3\left(1-x^{-1}\right)<x$ for any $x$, as the polynomial $x^{2}-3 x+3$ has no real roots.

Now we estimate $I_{2}$. We have

$$
I_{2}<\int_{n-1}^{\infty} \mathrm{d} s\left\{\int_{0}^{n}(t+s)^{-(q+\beta) / r} \mathrm{~d} t\right\}^{r}=n^{1+r-q-\beta} I_{3}
$$

where

$$
I_{3}=\int_{1-(1 / n)}^{\infty} \mathrm{d} s\left\{\int_{0}^{1}(t+s)^{-(q+\beta) / r} \mathrm{~d} t\right\}^{r} \leq \int_{1-(1 / n)}^{\infty} s^{-(q+\beta)} \mathrm{d} s<\infty
$$

since $q+\beta>1$. The required bound, $I_{2}=O\left(n^{r / \theta}\right)$, again follows from (4.3). This proves (3.1) for $1<r<\alpha$.

Now let $0<r \leq 1$. Then, from (4.1) and (4.2), we obtain $\mathrm{E}\left[\left|\sum_{t=1}^{n} X_{t}^{1}\right|^{r}\right] \leq C\left(\tilde{I}_{1}+\tilde{I}_{2}\right)$, where now

$$
\tilde{I}_{1}:=\sum_{|s| \leq n} \sum_{t=1}^{n} t^{-q-\beta}, \quad \tilde{I}_{2}:=\sum_{s \geq n} \sum_{t=1}^{n}(t+s)^{-q-\beta} .
$$


Since $\beta+q>1$, we have $\tilde{I}_{1}=O(n)$ and $\tilde{I}_{2}=O\left(n^{2-\beta-q}\right)$, and (3.1) follows from the fact that

$$
2-\beta-q<\frac{r(1+\alpha)}{\alpha(\beta+q)}
$$

for $r<\alpha$ sufficiently close to $\alpha$. As in the proof of (4.3), it is sufficient to prove (4.4) for $r=\alpha$, in which case it becomes $2-\beta-q<(1+\alpha) /(\beta+q)$, or $0<(\beta+q-1)^{2}+\alpha$. Lemma 3.1 is thus proved.

Proof of Lemma 3.2. The proof is very similar to that in Leipus and Surgailis (2003, p. 752) and is omitted.

The proof of Lemma 3.3 uses Lemmas 3.4, 3.5, and 4.1 and is postponed until the end of the section.

Lemma 4.1. Let $\tilde{\Phi} \geq 0$, let $\tilde{Z}$ and $\tilde{Z}^{0}$ be RVs with $\tilde{Z}^{0}$ independent of $\tilde{\Phi}$, and let

$$
\mathrm{P}[\tilde{\Phi}>u] \sim c_{0} u^{-\lambda / \alpha} \quad \text { as } u \rightarrow \infty,
$$

for some $c_{0}>0$ and $\lambda$ and $\alpha, 0<\lambda<\alpha<\infty$. Moreover, assume that there exist $r>\lambda$, $a$ nonrandom constant $C<\infty$, and a function $\delta(u), u>0$, with $\lim _{u \rightarrow \infty} \delta(u)=0$, such that

$$
\mathrm{P}\left[\left|\tilde{Z}^{0}\right|>x\right]+\mathrm{P}[|\tilde{Z}|>x \mid \tilde{\Phi}] \leq C x^{-r} \text { for all } x>0 \text {, almost surely, }
$$

and

$$
\sup _{x \in \mathbb{R}}\left|\mathrm{P}[\tilde{Z} \leq x \mid \tilde{\Phi}]-\mathrm{P}\left[\tilde{Z}^{0} \leq x\right]\right| \leq \delta(\tilde{\Phi}) \text { almost surely. }
$$

Let $\tilde{Y}:=\tilde{\Phi}^{1 / \alpha} \tilde{Z}$. Then

$$
\mathrm{P}[\tilde{Y}>x] \sim c_{1}^{+} x^{-\lambda} \quad \text { as } x \rightarrow \infty, \quad \mathrm{P}[\tilde{Y} \leq x] \sim c_{1}^{-}|x|^{-\lambda} \text { as } x \rightarrow-\infty,
$$

where

$$
c_{1}^{+}:=c_{0} \mathrm{E}\left[\left|\tilde{Z}^{0}\right|^{\lambda} \mathbf{1}\left(\tilde{Z}^{0}>0\right)\right], \quad c_{1}^{-}:=c_{0} \mathrm{E}\left[\left|\tilde{Z}^{0}\right|^{\lambda} \mathbf{1}\left(\tilde{Z}^{0}<0\right)\right] .
$$

Proof. Let $\tilde{X}:=\tilde{\Phi}^{1 / \alpha} \tilde{Z}^{0}$. The lemma follows from the facts that

$$
\mathrm{P}[\tilde{X}>x] \sim c_{1}^{+} x^{-\lambda} \quad \text { as } x \rightarrow \infty, \quad \mathrm{P}[\tilde{X} \leq x] \sim c_{1}^{-}|x|^{-\lambda} \quad \text { as } x \rightarrow-\infty,
$$

and

$\mathrm{P}[\tilde{Y}>x]-\mathrm{P}[\tilde{X}>x]=o\left(x^{-\lambda}\right), \quad \mathrm{P}[\tilde{Y} \leq-x]-\mathrm{P}[\tilde{X} \leq-x]=o\left(|x|^{-\lambda}\right), \quad$ as $x \rightarrow \infty$.

Relations (4.9) are well known (see Breiman (1965) and Pipiras et al. (2004)). Let us prove (4.10). We have

$$
\mathrm{P}[\tilde{Y}>x]=\mathrm{E}\left[\left.\mathrm{P}\left[\tilde{Z}>x u^{-1 / \alpha} \mid \tilde{\Phi}\right]\right|_{u=\tilde{\Phi}}\right], \quad \mathrm{P}[\tilde{X}>x]=\mathrm{E}\left[\left.\mathrm{P}\left[\tilde{Z}^{0}>x u^{-1 / \alpha}\right]\right|_{u=\tilde{\Phi}}\right],
$$

and, therefore,

$$
|\mathrm{P}[\tilde{Y}>x]-\mathrm{P}[\tilde{X}>x]| \leq \sum_{i=1}^{3} d_{i}(x),
$$


where

$$
\begin{aligned}
& d_{1}(x):=\mathrm{E}\left[\left|\mathrm{P}\left[\tilde{Z}>x u^{-1 / \alpha} \mid \tilde{\Phi}\right]\right|_{u=\tilde{\Phi}}-\left.\mathrm{P}\left[\tilde{Z}^{0}>x u^{-1 / \alpha}\right]\right|_{u=\tilde{\Phi}} \mid \mathbf{1}\left(x \tilde{\Phi}^{-1 / \alpha} \leq K\right)\right], \\
& d_{2}(x):=\mathrm{E}\left[\left.\mathrm{P}\left[\tilde{Z}>x u^{-1 / \alpha} \mid \tilde{\Phi}\right]\right|_{u=\tilde{\Phi}} \mathbf{1}\left(x \tilde{\Phi}^{-1 / \alpha}>K\right)\right], \\
& d_{3}(x):=\mathrm{E}\left[\left.\mathrm{P}\left[\tilde{Z}^{0}>x u^{-1 / \alpha}\right]\right|_{u=\tilde{\Phi}} \mathbf{1}\left(x \tilde{\Phi}^{-1 / \alpha}>K\right)\right] .
\end{aligned}
$$

Consider the last expression. As $\mathrm{P}\left[\tilde{Z}^{0}>x u^{-1 / \alpha}\right] \leq C u^{r / \alpha} / x^{r}$ by (4.6), we have

$$
\begin{aligned}
d_{3}(x) & \leq C x^{-r} \mathrm{E}\left[\tilde{\Phi}^{r / \alpha} \mathbf{1}\left(\tilde{\Phi} \leq(x / K)^{\alpha}\right)\right] \\
& =-C x^{-r} \int_{0}^{(x / K)^{\alpha}} u^{r / \alpha} \mathrm{dP}[\tilde{\Phi}>u] \\
& =C\left[-x^{-r}\left(\frac{x}{K}\right)^{r} \mathrm{P}\left[\tilde{\Phi}>\left(\frac{x}{K}\right)^{\alpha}\right]+x^{-r}\left(\frac{r}{\alpha}\right) \int_{0}^{(x / K)^{\alpha}} \mathrm{P}[\tilde{\Phi}>u] u^{(r / \alpha)-1} \mathrm{~d} u\right] \\
& \leq\left(C / K^{r-\lambda}\right) x^{-\lambda}
\end{aligned}
$$

by (4.5). We obtain the same bound for $d_{2}(x)$, also using (4.6) and (4.5). Therefore,

$$
\sup _{x>0} x^{\lambda}\left(d_{2}(x)+d_{3}(x)\right)
$$

can be made arbitrarily small by choosing $K>0$ to be large enough.

Let us estimate $d_{1}(x)$. As $\lim _{u \rightarrow \infty} \delta(u) \rightarrow 0$, for any $K<\infty$ and $\delta_{0}>0$ there exists a $u_{0} \equiv u_{0}\left(K, \delta_{0}\right)>0$ such that $\delta(u)<\delta_{0} / K^{\lambda}$ for all $u>u_{0}$. Then, by (4.7),

$$
\sup _{v \in \mathbb{R}}\left|\mathrm{P}[\tilde{Z}>v \mid \tilde{\Phi}]-\mathrm{P}\left[\tilde{Z}^{0}>v\right]\right|<\delta_{0} / K^{\lambda}, \quad \text { almost surely on }\left\{\tilde{\Phi}>u_{0}\right\} .
$$

Then, for all $x>u_{0}^{1 / \alpha} K$ large enough,

$$
d_{1}(x) \leq \frac{\delta_{0}}{K^{\lambda}} \mathrm{P}\left[\tilde{\Phi} \geq\left(\frac{x}{K}\right)^{\alpha}\right] \leq \frac{C \delta_{0} / K^{\lambda}}{(x / K)^{\lambda}} \leq C \delta_{0} x^{-\lambda} .
$$

Hence, $\lim \sup _{x \rightarrow \infty} x^{\lambda}\left|d_{1}(x)\right| \leq C \delta_{0}$, thereby proving the first relation of (4.10). The second relation of (4.10) follows similarly.

Proof of Lemma 3.4. Let us consider

$$
\Theta_{n}(v):=\frac{1}{n^{1+\alpha}} \Phi\left(1-\frac{v}{n}, n\right)=\frac{1}{v^{\alpha} n} \sum_{j=1}^{n}\left(1-\left(\left(1-\frac{v}{n}\right)^{n}\right)^{j / n}\right)^{\alpha}, \quad 0 \leq v \leq n .
$$

Since $(1-(v / n))^{n} \rightarrow \mathrm{e}^{-v}$, by the dominated convergence theorem $\Theta_{n}(v)$ converges to $\Theta(v)$, in (3.8), for each $v>0$. We note the bound

$$
\Theta_{n}(v) \leq C /(1+v)^{\alpha} .
$$

Indeed, for $v>1$, (4.11) follows by writing $\Phi(a, n) \leq n /(1-a)^{\alpha}$ and from the definition of $\Theta_{n}(v)$. For $v \leq 1,(4.11)$ follows trivially from $\Phi(a, \bar{n}) \leq n^{1+\alpha}$. 
To prove (3.6), first consider the case $q>0$. Then $\mathrm{P}[\Phi(A, U)>x]=p_{0}(x)+p_{1}(x)$, where

$$
\begin{aligned}
p_{0}(x) & :=\mathrm{P}\left[\Phi(A, U)>x, 1-\epsilon<A<1, U>n_{0}\right] \\
p_{1}(x) & :=\mathrm{P}\left[\Phi(A, U)>x, A \leq 1-\epsilon \text { or } U \leq n_{0}\right] \\
& \leq \mathrm{P}[\Phi(A, U)>x, A \leq 1-\epsilon]+\mathrm{P}\left[\Phi(A, U)>x, U \leq n_{0}\right]
\end{aligned}
$$

Relation (3.6) follows from

$$
\lim _{n_{0} \rightarrow \infty, \epsilon \rightarrow 0} \limsup _{x \rightarrow \infty}\left|\frac{p_{0}(x)}{c_{V} x^{-(\beta+q) /(1+\alpha)}}-1\right|=0
$$

and the fact that, for any fixed $n_{0}<\infty$ and $\epsilon>0$,

$$
\lim _{x \rightarrow \infty} p_{1}(x) x^{\lambda / \alpha}=0 .
$$

Let us prove (4.12). As in Leipus and Surgailis (2003),

$$
p_{0}(x)=c_{V}(x) x^{-\lambda / \alpha}\left(1+\delta\left(n_{0}, \epsilon\right)\right),
$$

where $\delta\left(n_{0}, \epsilon\right) \rightarrow 0$ as $n_{0} \rightarrow \infty$ and $\epsilon \rightarrow 0$, and where

$$
\begin{aligned}
c_{V}(x):=c_{U} f_{1} \int_{0}^{\infty} \frac{\mathrm{d} y}{y^{1+\beta+q}} \omega_{1}(x ; y) \int_{0}^{\infty} \frac{\mathrm{d} v}{v^{1-q}} \omega_{2}(x ; v, y) \\
\times \mathbf{1}\left(y^{1+\alpha} \Theta_{\left[x^{1 /(1+\alpha)} y\right]}(v)>\omega_{3}(x ; y)\right) .
\end{aligned}
$$

The functions $\omega_{i}, i=1,2,3$, are uniformly bounded in all arguments and tend to 1 for any $v, y>0$ as $x \rightarrow \infty$. Then (4.12) follows from

$$
\lim _{x \rightarrow \infty} c_{V}(x)=c_{V}
$$

Furthermore, using (4.11),

$$
c_{V}(x) \leq \bar{c}_{V}:=C \int_{0}^{\infty} \frac{\mathrm{d} y}{y^{1+\beta+q}} \int_{0}^{\infty} \frac{\mathrm{d} v}{v^{1-q}} \mathbf{1}\left(C \frac{y^{1+\alpha}}{(1+v)^{\alpha}}>1\right),
$$

which is finite, since $1+\beta+q>1$ and $\beta+q-q(1-\alpha) / \alpha>0$, or $\beta>q / \alpha$. Note that the last inequality follows from $\beta+q<1+\alpha$ and $\beta>1$, as these imply that $q<\alpha$ and, therefore, that $\beta>1>q / \alpha$. Therefore (4.14) holds by the dominated convergence theorem, proving (4.12).

To finish the proof of (3.6) in the case $q>0$, we need to prove (4.13), where now $\epsilon>0$ and $n_{0}<\infty$ are fixed. Note that $\Phi(a, n) \leq C n$ for $a \leq 1-\epsilon$; therefore,

$$
\mathrm{P}[\Phi(A, U)>x, A \leq 1-\epsilon] \leq \mathrm{P}\left[U>C^{-1} x\right] \leq C x^{-\beta}=o\left(x^{-\lambda / \alpha}\right),
$$

as $\beta>1>\lambda / \alpha$. On the other hand, $\mathrm{P}\left[\Phi(A, U)>x, U \leq n_{0}\right]=0$ for sufficiently large $x$, since $\Phi(a, n)$ is bounded by $n_{0}^{\alpha+1}$ for $n \leq n_{0}$ and $a \in[0,1]$ (see (3.4)).

If $q=0$ then $\mathrm{P}[\Phi(A, U)>x]=f_{1} P[\Phi(1, U)>x]+P[\Phi(A, U)>x, A<1]$. As $\Phi(1, n)=\sum_{k=1}^{n} k^{\alpha} \sim n^{1+\alpha} /(1+\alpha)$, we have

$$
\begin{aligned}
\mathrm{P}[\Phi(1, U)>x] & \sim \mathrm{P}\left[U^{1+\alpha}>(1+\alpha) x\right] \\
& =\mathrm{P}\left[U>((1+\alpha) x)^{1 /(1+\alpha)}\right] \\
& \sim\left(c_{V} / f_{1}\right) x^{-\lambda / \alpha} \text { as } x \rightarrow \infty,
\end{aligned}
$$


with $c_{V}$ as given in (3.7). It remains to show that

$$
\limsup _{x \rightarrow \infty} x^{\lambda / \alpha} \mathrm{P}[\Phi(A, U)>x, A<1]=0 .
$$

For any $\delta>0$, we can find a $\delta^{\prime}>0$ such that $\mathrm{P}\left[1-\delta^{\prime}<A<1\right]<\delta$. Then, using $\Phi(a, n) \leq \Phi(1, n)$, we have

$$
\mathrm{P}\left[\Phi(A, U)>x, 1-\delta^{\prime}<A<1\right] \leq \delta \mathrm{P}[\Phi(1, U)>x] \leq C \delta x^{-\lambda / \alpha},
$$

according to the argument above. Finally, for any fixed $\delta^{\prime}>0$,

$$
\sup _{0 \leq a \leq 1-\delta^{\prime}} \Phi(a, n) \leq\left(1-a^{n+1}\right)\left(\delta^{\prime}\right)^{-\alpha} n \leq C n
$$

and, therefore,

$$
\mathrm{P}\left[\Phi(A, U)>x, A \leq 1-\delta^{\prime}\right] \leq \mathrm{P}[C U>x] \leq C x^{-\beta}=o\left(x^{-\lambda / \alpha}\right), \quad \beta>1>\lambda / \alpha .
$$

As $\delta>0$ is arbitrary, this proves (4.15) and, thus, the lemma.

Proof of Lemma 3.5. First let $0<\alpha<2$, with $\alpha \neq 1$. The proof of (3.9) given below uses the bound of the rate of convergence in the central limit theorem for sums of independent RVs in the domain of attraction of an $\alpha$-stable law obtained in Paulauskas (1974). To that end, let

$$
S(a, n):=\sum_{i=1}^{n} b_{n, i} \zeta_{i}, \quad T(a, n)=\sum_{i=1}^{n} b_{n, i} \varepsilon_{i}, \quad b_{n, i}:=1+a+\cdots+a^{n-i},
$$

where $\zeta_{i}, i=1,2, \ldots$, are independent copies of the RV $Z$ in (1.4). Note that $S(a, n)$ is a weighted sum of $\alpha$-stable RVs and that the normalized RV $S(a, n) / \Phi^{1 / \alpha}(a, n)$ has the same distribution as the RV $Z$ in (3.9). Let

$$
\begin{aligned}
\Delta(a, n) & :=\sup _{x \in \mathbb{R}}|\mathrm{P}[Z(a, n) \leq x]-\mathrm{P}[Z \leq x]| \\
& =\sup _{x \in \mathbb{R}}\left|\mathrm{P}\left[\frac{T(a, n)}{\Phi^{1 / \alpha}(a, n)} \leq x\right]-\mathrm{P}\left[\frac{S(a, n)}{\Phi^{1 / \alpha}(a, n)} \leq x\right]\right| .
\end{aligned}
$$

Let $r:=\alpha+\delta$, where $\delta>0$ is the same as in Assumption $\mathrm{D}(\alpha, \delta)$. Observe that $\Phi(a, n)=$ $\sum_{i=1}^{n} b_{n, i}^{\alpha}$, and that the absolute pseudomoment obeys $\mu_{r}\left(b_{n, i} \varepsilon_{i}\right)=b_{n, i}^{r} \mu_{r}(\varepsilon)<\infty$. Let

$$
\begin{aligned}
L_{n, r}(a) & :=\sum_{i=1}^{n} \frac{\mu_{r}\left(b_{n, i} \varepsilon_{i}\right)}{\Phi^{r / \alpha}(a, n)}=\mu_{r}(\varepsilon) \frac{\sum_{i=1}^{n} b_{n, i}^{r}}{\left(\sum_{i=1}^{n} b_{n, i}^{\alpha}\right)^{r / \alpha}}, \\
\gamma_{n}(a) & :=\frac{\max _{1 \leq i \leq n} b_{n, i}}{\left(\sum_{i=1}^{n} b_{n, i}^{\alpha}\right)^{1 / \alpha}} .
\end{aligned}
$$

According to the bound of Paulauskas (1974, Theorem 1) (see also Christoph and Wolf (1992, p. 59)),

$$
\Delta(a, n) \leq K_{\alpha} \max \left\{L_{n, r}(a),\left(L_{n, r}(a)\right)^{1 /(r+1)} \gamma_{n}^{r /(r+1)}(a)\right\},
$$

where $K_{\alpha}$ is an absolute constant depending only on $\alpha$. Clearly, $0 \leq \gamma_{n}(a) \leq 1$ and (3.9) will follow from (4.16) if we can show that

$$
\sup _{0 \leq a \leq 1} L_{n, r}(a) \rightarrow 0 \quad \text { as } n \rightarrow \infty .
$$


According to Lemma 4.2, below, the function $L_{n, r}(a)$ is nondecreasing in $a \in[0,1]$ for any $n \geq 1$. Therefore, (4.17) follows from

$$
L_{n, r}(1)=\mu_{r}(\varepsilon) \frac{\sum_{i=1}^{n} i^{r}}{\left(\sum_{i=1}^{n} i^{\alpha}\right)^{r / \alpha}}=O\left(n^{-(r-\alpha) / \alpha}\right)=o(1),
$$

as $r>\alpha$. This proves (3.9) for $0<\alpha<2$.

The case in which $\alpha=2$ is simpler. Let $r=2+\delta$, again with $\delta$ as in Assumption $\mathrm{D}(\alpha, \delta)$ (ii). In this case, $Z$ is a Gaussian $\mathrm{RV}$ with mean 0 and variance $\mathrm{E}\left[\varepsilon^{2}\right]$, and $\Phi(a, n)=$ $\operatorname{var}(T(a, n)) / \mathrm{E}\left[\varepsilon^{2}\right]$. By applying a standard estimate of the rate of convergence in the central limit theorem for independent summands with finite $r$ th moment (Petrov (1995, p. 151)), we obtain $\Delta(a, n) \leq K \tilde{L}_{n, r}(a)$, where $K$ is an absolute constant and

$$
\tilde{L}_{n, r}(a):=\frac{\mathrm{E}\left[|\varepsilon|^{r}\right] \sum_{i=1}^{n} b_{n, i}^{r}}{\left(\mathrm{E}\left[\varepsilon^{2}\right] \sum_{i=1}^{n} b_{n, i}^{2}\right)^{r / 2}} .
$$

Again, using Lemma 4.2 and noting that

$$
\tilde{L}_{n, r}(1)=\frac{\mathrm{E}\left[|\varepsilon|^{r}\right] \sum_{i=1}^{n} i^{r}}{\left(\mathrm{E}\left[\varepsilon^{2}\right] \sum_{i=1}^{n} i^{2}\right)^{r / 2}}=O\left(n^{-\delta / 2}\right),
$$

we obtain (3.9) in the case $\alpha=2$.

Let us now prove (3.10). Write $Z(a, n)=\sum_{i=1}^{n} \beta_{n, i} \varepsilon_{i}$, where

$$
\beta_{n, i}:=\frac{b_{n, i}}{\Phi^{1 / \alpha}(a, n)}, \quad \sum_{i=1}^{n}\left|\beta_{n, i}\right|^{\alpha}=1 .
$$

Let $\hat{\varepsilon}_{i}, i \geq 1$, be independent copies of $\varepsilon_{i}, i \geq 1$, and let $\hat{Z}(a, n):=\sum_{i=1}^{n} \beta_{n, i} \hat{\varepsilon}_{i}$. Then $\tilde{\varepsilon}_{i}:=$ $\varepsilon_{i}-\hat{\varepsilon}_{i}, i \geq 1$, are symmetric, i.i.d. RVs and

$$
\tilde{Z}(a, n):=Z(a, n)-\hat{Z}(a, n)=\sum_{i=1}^{n} \beta_{n, i} \tilde{\varepsilon}_{i}
$$

also has a symmetric distribution. Let $H>0$ satisfy

$$
\mathrm{P}[|Z(a, n)|>H] \leq \frac{1}{2}
$$

Then

$$
\mathrm{P}[|Z(a, n)|>x] \leq 2 \mathrm{P}[|\tilde{Z}(a, n)|>x-H] .
$$

Introduce the Lorentz norm

$$
\|\zeta\|_{\alpha, \infty}:=\left(\sup _{x>0} x^{\alpha} \mathrm{P}[|\zeta|>x]\right)^{1 / \alpha} .
$$

We shall use the following inequality, due to Rosiński (1980, Theorem 1). Let $0<\alpha \leq 2$ and let $\xi_{i}, i=1,2, \ldots$, be independent symmetric RVs such that $\left\|\xi_{i}\right\|_{\alpha, \infty}<\infty, i \geq 1$. Then, for any $n \geq 1$,

$$
\left\|\sum_{i=1}^{n} \xi_{i}\right\|_{\alpha, \infty}^{\alpha} \leq C_{\alpha} \sum_{i=1}^{n}\left\|\xi_{i}\right\|_{\alpha, \infty}^{\alpha}
$$


where $C_{\alpha}$ is an absolute constant depending on $\alpha$ only. Applying (4.20) to the sum $\tilde{Z}(a, n)$, yields

$$
\begin{aligned}
\mathrm{P}[|\tilde{Z}(a, n)|>x] & \leq x^{-\alpha}\|\tilde{Z}(a, n)\|_{\alpha, \infty}^{\alpha} \\
& \leq C_{\alpha} x^{-\alpha} \sum_{i=1}^{n}\left\|\beta_{n, i} \tilde{\varepsilon}_{i}\right\|_{\alpha, \infty}^{\alpha} \\
& \leq C_{\alpha}\left\|\tilde{\varepsilon}_{1}\right\|_{\alpha, \infty}^{\alpha} x^{-\alpha} \sum_{i=1}^{n}\left|\beta_{n, i}\right|^{\alpha} \\
& =\tilde{C}_{\alpha} x^{-\alpha},
\end{aligned}
$$

where $\tilde{C}_{\alpha}=C_{\alpha}\left\|\tilde{\varepsilon}_{1}\right\|_{\alpha, \infty}^{\alpha}$ does not depend on $n$, a, or $x$. Clearly, (3.10) follows from (4.21) and (4.19), provided that we can find an $H>0$ that satisfies (4.18) and is independent of $n$ and $a$ for all $n \geq n_{0}$ large enough. The last fact follows from (3.9), or (4.16) and (4.17). Indeed, as $\mathrm{P}[|Z(a, n)|>H] \leq \mathrm{P}[|Z|>H]+2 \Delta(a, n)$, we can choose $H$ and $n_{0}$ to be large enough that both $\mathrm{P}[|Z|>H]<\frac{1}{4}$ and $\sup _{a \in[0,1]} \Delta(a, n)<\frac{1}{8}, n \geq n_{0}$. Lemma 3.5 is thus proved.

Lemma 4.2. Let

$$
L_{n}(a):=\frac{\zeta(a, n, r)}{\zeta^{r / \alpha}(a, n, \alpha)}, \quad \zeta(a, n, r):=\sum_{i=1}^{n}\left(1-a^{i}\right)^{r} .
$$

Then for any $n \geq 1, a \in[0,1]$, and any $\alpha, r \geq \alpha>0$, we have $\partial L_{n}(a) / \partial a \geq 0$.

Proof. It suffices to prove the lemma for $a \in(0,1)$. We have

$$
\frac{\partial L_{n}(a)}{\partial a}=\frac{1}{\zeta^{r / \alpha}(a, n, \alpha)}\left[\zeta_{a}^{\prime}(a, n, r)-\frac{r}{\alpha} \zeta_{a}^{\prime}(a, n, \alpha) \frac{\zeta(a, n, r)}{\zeta(a, n, \alpha)}\right],
$$

where

$$
\zeta_{a}^{\prime}(a, n, r)=-\sum_{i=1}^{n} r\left(1-a^{i}\right)^{r-1} i a^{i-1}
$$

To prove the lemma, it suffices to show that the expression in square brackets is nonnegative, i.e. that

$$
\frac{\zeta_{a}^{\prime}(a, n, r)}{\zeta_{a}^{\prime}(a, n, \alpha)} \leq \frac{r}{\alpha} \frac{\zeta(a, n, r)}{\zeta(a, n, \alpha)}
$$

or

$$
\frac{\sum_{i=1}^{n}\left(1-a^{i}\right)^{r-1} i a^{i-1}}{\sum_{i=1}^{n}\left(1-a^{i}\right)^{\alpha-1} i a^{i-1}} \leq \frac{\sum_{i=1}^{n}\left(1-a^{i}\right)^{r}}{\sum_{i=1}^{n}\left(1-a^{i}\right)^{\alpha}} .
$$

We shall prove (4.22) by induction on $n$. For $n=1$, it becomes the identity, $(1-a)^{r-\alpha}=$ $(1-a)^{r-\alpha}$, which is valid for any $a, 0 \leq a \leq 1$.

Let us demonstrate the induction step $n \rightarrow n+1$, or the inequality

$$
\frac{\sum_{i=1}^{n+1}\left(1-a^{i}\right)^{r-1} i a^{i-1}}{\sum_{i=1}^{n+1}\left(1-a^{i}\right)^{\alpha-1} i a^{i-1}} \leq \frac{\sum_{i=1}^{n+1}\left(1-a^{i}\right)^{r}}{\sum_{i=1}^{n+1}\left(1-a^{i}\right)^{\alpha}}
$$


To this end, let

$$
\begin{array}{ll}
A:=\sum_{i=1}^{n}\left(1-a^{i}\right)^{r-1} i a^{i-1}, & B:=\sum_{i=1}^{n}\left(1-a^{i}\right)^{\alpha-1} i a^{i-1}, \\
C:=\sum_{i=1}^{n}\left(1-a^{i}\right)^{r}, & D:=\sum_{i=1}^{n}\left(1-a^{i}\right)^{\alpha},
\end{array}
$$

whence (4.22) becomes $A / B \leq C / D$, while (4.23) can be written as

$$
\frac{A+\left(1-a^{n+1}\right)^{r-1}(n+1) a^{n}}{B+\left(1-a^{n+1}\right)^{\alpha-1}(n+1) a^{n}} \leq \frac{C+\left(1-a^{n+1}\right)^{r}}{D+\left(1-a^{n+1}\right)^{\alpha}} .
$$

Using $A D \leq B C,(4.24)$ is implied by the following inequality:

$$
\begin{aligned}
& A\left(1-a^{n+1}\right)^{\alpha}+D\left(1-a^{n+1}\right)^{r-1}(n+1) a^{n} \\
& \quad \leq B\left(1-a^{n+1}\right)^{r}+C\left(1-a^{n+1}\right)^{\alpha-1}(n+1) a^{n} .
\end{aligned}
$$

By substituting the full expressions for $A, B, C$, and $D$ into this inequality and using elementary transformations, it becomes

$$
\begin{aligned}
& \sum_{i=1}^{n}\left[\left(1-a^{n+1}\right)^{r-\alpha}-\left(1-a^{i}\right)^{r-\alpha}\right]\left(1-a^{i}\right)^{\alpha-1}\left[\left(1-a^{n+1}\right) i a^{i-1}-(n+1) a^{n}\left(1-a^{i}\right)\right] \\
& \quad \geq 0 .
\end{aligned}
$$

Note that each term of this sum is nonnegative. Indeed, $\left(1-a^{n+1}\right)^{r-\alpha}-\left(1-a^{i}\right)^{r-\alpha} \geq 0$ and $\left(1-a^{i}\right)^{\alpha-1} \geq 0$ for any $a \in[0,1], i$ with $1 \leq i \leq n$, and $r \geq \alpha$. It remains to show that

$$
\left(1-a^{n+1}\right) i a^{i-1}-(n+1) a^{n}\left(1-a^{i}\right) \geq 0 .
$$

The left-hand side of (4.26) can be written as $a^{i-1} h(a)$, with

$$
h(a):=(n+1-i) a^{n+1}+i-(n+1) a^{n+1-i} .
$$

Note that $h(1)=0$ and

$$
\begin{aligned}
h^{\prime}(a) & =(n+1)(n+1-i) a^{n}-(n+1)(n+1-i) a^{n-i} \\
& =(n+1)(n+1-i)\left(a^{n}-a^{n-i}\right) \\
& \leq 0
\end{aligned}
$$

for $a \in[0,1]$ and $i \leq n+1$. Therefore, $h(a) \geq 0$ for $a \in[0,1]$. This proves (4.26), (4.25), and the induction step $n \rightarrow n+1$. Lemma 4.2 is thus proved.

Proof of Lemma 3.3. We use Lemma 4.1 with $\tilde{Z}:=Z(A, U)$ and $\tilde{\Phi}:=\Phi(A, U)$. Condition (4.5) follows from Lemma 3.4 and conditions (4.6) and (4.7) follow from Lemma 3.5. Thus, (4.8) holds and, in turn, implies the tail behavior of $Y$. 
Let us now prove (3.5). Choose $\lambda=1$ and an $r$ such that $1<r<\alpha$. Using $\mathrm{E}[\varepsilon]=0$, $\mathrm{E}\left[|\varepsilon|^{r}\right]<\infty, 0 \leq A \leq 1$, the inequality $|\sin (x)-x|<2|x|^{r}(1<r<2)$, and (2.3), we obtain

$$
\begin{aligned}
\left|n \mathrm{E}\left[\sin \left(\frac{Y}{n}\right)\right]\right| & =n\left|\mathrm{E}\left[\mathrm{E}\left[n^{-1} \sum_{i=1}^{U} A^{i-1} \varepsilon_{i}-\sin \left(n^{-1} \sum_{i=1}^{U} A^{i-1} \varepsilon_{i}\right) \mid A, U\right]\right]\right| \\
& \leq 2 n^{1-r} \mathrm{E}\left[\mathrm{E}\left[\left|\sum_{i=1}^{U} A^{i-1} \varepsilon_{i}\right|^{r} \mid A, U\right]\right] \\
& \leq 4 n^{1-r} \mathrm{E}\left[|\varepsilon|^{r}\right] \mathrm{E}\left[\sum_{i=1}^{U} A^{r(i-1)}\right] \\
& \leq 4 n^{1-r} \mathrm{E}\left[|\varepsilon|^{r}\right] \mathrm{E}[U] \\
& =o(1),
\end{aligned}
$$

as $r>1$. This proves (3.5), completing the proof of Lemma 3.3.

\section{Acknowledgement}

The authors are deeply obliged to an anonymous referee whose numerous comments and suggestions helped to considerably improve the first version of the paper.

\section{References}

Blanchard, O. J. (1979). Speculative bubbles, crashes and rational expectations. Econom. Lett. 3, 387-389.

Brandt, A. (1986). The stochastic equation $Y_{n+1}=A_{n} Y_{n}+B_{n}$ with stationary coefficients. Adv. Appl. Prob. 18, 211-220.

Breiman, L. (1965). On some limit theorems similar to the arc-sin law. Theory Prob. Appl. 10, 323-331.

Chistyakov, G. P. and Götze, F. (2004). Limit distributions of Studentized means. Ann. Prob. 32, 28-77.

Christoph, G. And Wolf, W. (1992). Convergence Theorems with a Stable Limit Law (Math. Res. 70). AkademieVerlag, Berlin.

Cox, D. R. (1984). Long-range dependence: a review. In Statistics: an Appraisal, eds H. A. David and H. T. David, Iowa State University Press, pp. 55-74.

Davidson, J. AND SibBertsen, P. (2005). Generating schemes for long memory processes: regimes, aggregation and linearity. J. Econometrics 128, 253-282.

Dehling, H. AND Philipp, W. (2002). Empirical process techniques for dependent data. In Empirical Process Techniques for Dependent Data, eds H. Dehling, T. Mikosch and M. Sørensen, Birkhäuser, Boston, MA, pp. 3-113.

Diebold, F. X. AND Inoue, A. (2001). Long memory and regime switching. J. Econometrics 105, 131-159.

Feller, W. (1966). An Introduction to Probability Theory and Its Applications, Vol. 2. John Wiley, New York.

Gourieroux, C. AND JASIAK, J. (2001). Memory and infrequent breaks. Econom. Lett. 70, 29-41.

Granger, C. W. J. AND Hyung, N. (2004). Occasional structural breaks and long memory, with application to the S\&P 500 absolute stock returns. J. Emp. Finance 11, 399-421.

Heyde, C. C. And Yang, Y. (1997). On defining long-range dependence. J. Appl. Prob. 34, 939-944.

JENSEN, M. J. AND LiU, M. (2006). Do long swings in the business cycle lead to strong persistence in output? J. Monetary Econom. 53, 597-611.

KARLSEN, H. A. (1990). Existence of moments in a stationary difference equation. Adv. Appl. Prob. 22, $129-146$.

LeIPUs, R. AND Surgailis, D. (2003). Random coefficient autoregression, regime switching and long memory. Adv. Appl. Prob. 35, 737-754.

Leipus, R. And Viano, M.-C. (2003). Long memory and stochastic trend. Statist. Prob. Lett. 61, 177-190.

Leipus, R., Paulauskas, V. and Surgailis, D. (2005). Renewal regime switching and stable limit laws. J. Econometrics 129, 299-327.

LIU, M. (2000). Modeling long memory in stock market volatility. J. Econometrics 99, 139-171.

Mikosch, T. AND STĂRICĂ, C. (2004). Nonstationarities in financial time series, the long-range dependence and the IGARCH effects. Rev. Econom. Statist. 86, 378-390.

Mikosch, T., Resnick, S., Rootzén, H. and Stegeman, A. (2002). Is network traffic approximated by stable Lévy motion or fractional Brownian motion? Ann. Appl. Prob. 12, 23-68. 
PARKe, W. R. (1999). What is fractional integration? Rev. Econom. Statist. 81, 632-638.

Paulauskas, V. (1974). The estimation of the remainder term in limit theorem with a limiting stable distribution. Lithuanian Math. J. 14, 165-187.

Petrov, V. V. (1975). Sums of Independent Random Variables. Springer, New York.

Pipiras, V., Taqqu, M. S. and Levy, J. B. (2004). Slow, fast and arbitrary growth conditions for renewal reward processes when both the renewals and the rewards are heavy-tailed. Bernoulli 10, 121-163.

Pourahmadi, M. (1988). Stationarity of the solution of $X_{t}=A_{t} X_{t-1}+\varepsilon_{t}$ and analysis of non-Gaussian dependent variables. J. Time Ser. Anal. 9, 225-239.

Rosiński, J. (1980). Remarks on Banach spaces of stable type. Prob. Math. Statist. 1, 67-71.

Samorodnitsky, G. and Taqqu, M. S. (1994). Stable Non-Gaussian Random Processes. Chapman and Hall, New York.

TAQQU, M. S. AND LEVY, J. B. (1986). Using renewal processes to generate long-range dependence and high variability. In Dependence in Probability and Statistics, eds E. Eberlein and M. S. Taqqu, Birkhäuser, Boston, MA, pp. 73-89.

VERVAAT, W. (1979). On a stochastic difference equation and a representation of nonnegative infinitely divisible random variables. Adv. Appl. Prob. 11, 750-783.

VON BAHR, B. AND EsSEen, C.-G. (1965). Inequalities for the $r$ th absolute moment of a sum of random variables, $1 \leq r \leq 2$. Ann. Math. Statist. 36, 299-303. 